\title{
Research regarding use of pneumatic linear actuator
}

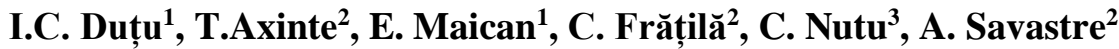 \\ ${ }^{1}$ University Politehnica of Bucharest, Romania \\ ${ }^{2}$ Research and Innovation Center for Navy, Constanța, Romania \\ ${ }^{3}$ Constanta Maritime University, Romania
}

Corresponding author: T. Axinte, tibi_axinte@yahoo.com

\begin{abstract}
This article represents research regarding use of pneumatic linear actuator. A pneumatic linear drive is a device that creates motion in a straight line. The linear drive with solenoid in this manuscript is a pneumatic actuator. The pneumatic linear drive works only with compressed air. Compressed air usually has a pressure of between $2 \cdot 10^{5} \mathrm{~Pa}$ and $6 \cdot 10^{5} \mathrm{~Pa}$. Linear drive with solenoid can be used for various applications, including pulling/pushing, pick-andplace applications or lifting/moving. After Introduction, the authors study two pneumatic circuits using linear drive with solenoid coupling. In fact, the first pneumatic scheme in this paper has only one device (linear drive 1-1). But, the second pneumatic circuit has two actuators (linear drives 2-1 and 2-2). Further on, the authors present two electro-pneumatic circuits. In any case, a simple electro-pneumatic circuit that has a linear drive with solenoid coupling. Moreover, the second electro-pneumatic system has a linear drive with solenoid coupling and PLC (Programmable Logic Controller). The pneumatic and electro-pneumatic circuits given in this manuscript are made using FluidSim software from Festo.
\end{abstract}

Keywords. pneumatic, scheme, linear, actuator, device.

\section{Introduction}

A pneumatic linear actuator is a device that creates motion only in a straight line, in contrast to circular motion of a pneumatic motor (air motor).

Various types of linear actuators pneumatic are used in the specific installations, [1].

The types of linear actuator are used depending on the functions of the circuits. These devices are mounted on both pneumatic and electro-pneumatic circuits.

In practice, there are used various types of pneumatic linear drive:

$>$ Pneumatic linear actuator with solenoid coupling, Figure 1.

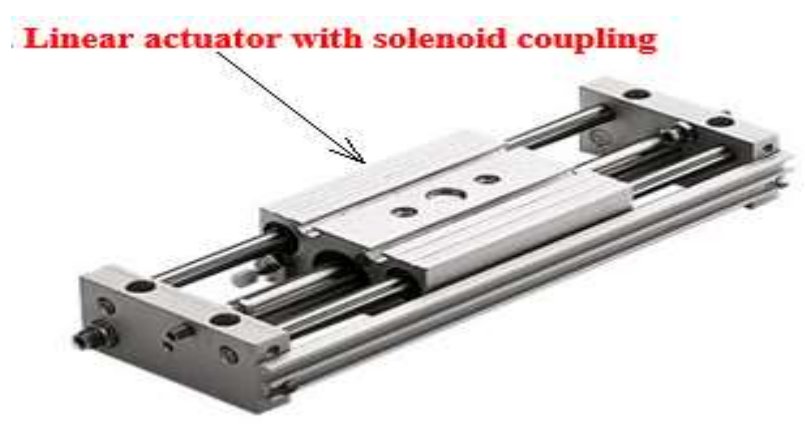

Figure 1. Linear actuator with solenoid coupling 
In linear drive with solenoid coupling, the sliding of the piston in the double rod cylinder is controlled by a reciprocal input of compressed air, [2].

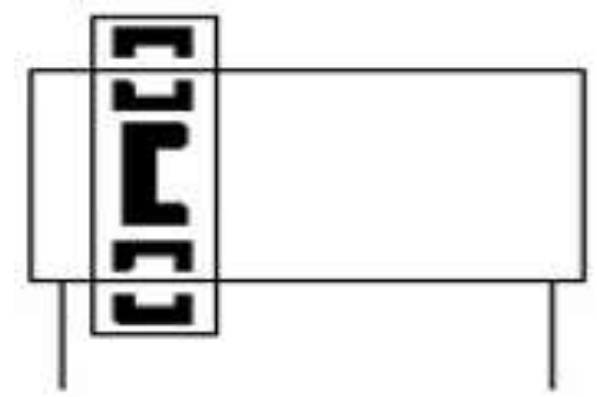

Figure 2. Symbol of first linear drive with solenoid coupling

The linear actuator with solenoid coupling is represented by a specific standard symbol, Figure 2 .

Pneumatic linear actuator with shape-fitting adaptor

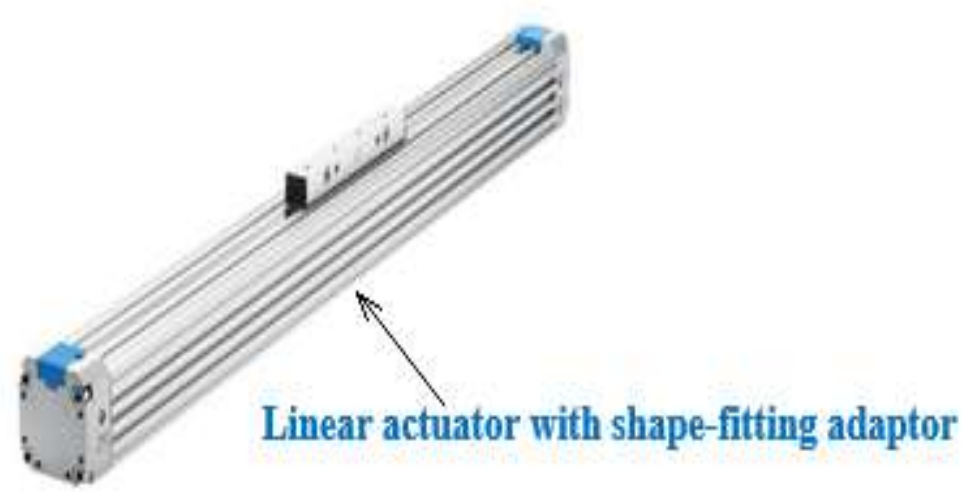

Figure 3. Linear actuator with shape-fitting adaptor

In linear actuator with shape-fitting adaptor, the sledge of the double acting cylinder without a piston rod is controlled by alternating the compressed air input, Figure. 3.

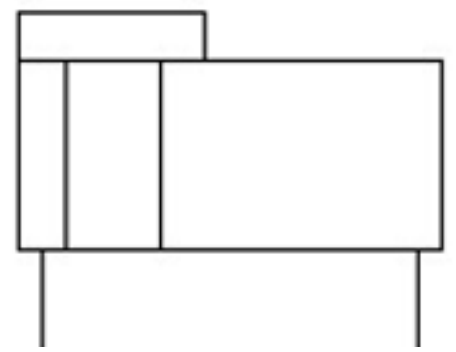

Figure 4. Symbol of second linear drive shape-fitting adaptor

In scheme, the pneumatic linear actuator with shape-fitting adaptor has a specific symbol, Fig. 4.

\section{The pneumatic circuits with linear actuator}

The pneumatic circuits with linear drive has a very simple construction in this paper.

A linear drive delivered from this pneumatic installation is often used in areas of extreme temperatures due to the safety of using air rather electricity or hazardous chemicals, [5].

Besides, the pneumatic installation with linear drive is a low-cost option.

The following pneumatic scheme presented in this article operates only when to $4 / 3$ way air directional valve will be given a manual command, using SI. 
In the table 1 below there are given the component devices used in the first pneumatic scheme.

\begin{tabular}{l|c}
\hline \multicolumn{1}{c|}{ Description } & Number of components \\
\hline Compressed air supply & 1 \\
\hline Air Filter & 1 \\
\hline 4/3 way directional valve & 1 \\
\hline Throttle valve & 2 \\
\hline Linear drive with shape-fitting adaptor & 1 \\
\hline
\end{tabular}

Table 1. Devices in the first pneumatic circuit

The first pneumatic scheme studied by authors has one linear drive 1-1, Figure 5.

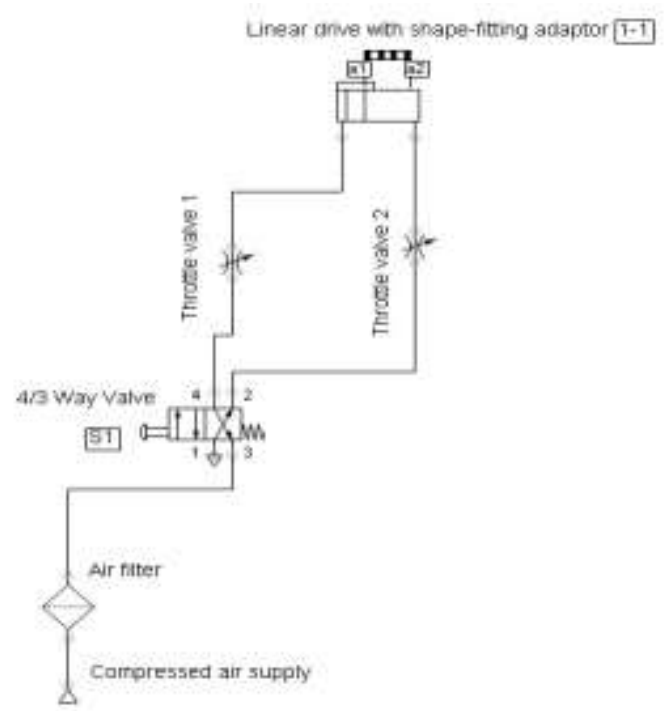

Figure 5. First pneumatic circuit using one linear actuator

The first pneumatic circuit operates if the operator presses $S 1$ button of the $4 / 3$ way directional valve with spring. Then, the piston of linear drive (1-1) moves from point a1 to point a2. After that, the piston returns from point a2 to point a1, because the 4/3 way directional valve has a spring, Figure 6 .

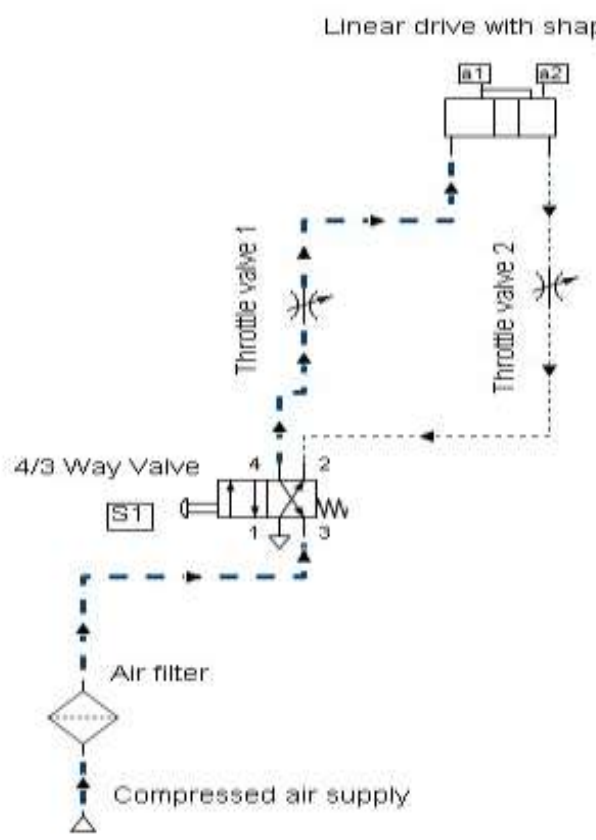

Figure 6. Simulation of the first pneumatic circuit 
The diagrams given show variation of the following functional parameters of the linear drive with shapefitting adaptor, Figure 7:

- Position $-\mathrm{x}[\mathrm{mm}]$;

- Velocity - v[m/s];

- Acceleration $-\left[\mathrm{m} / \mathrm{s}^{2}\right]$.

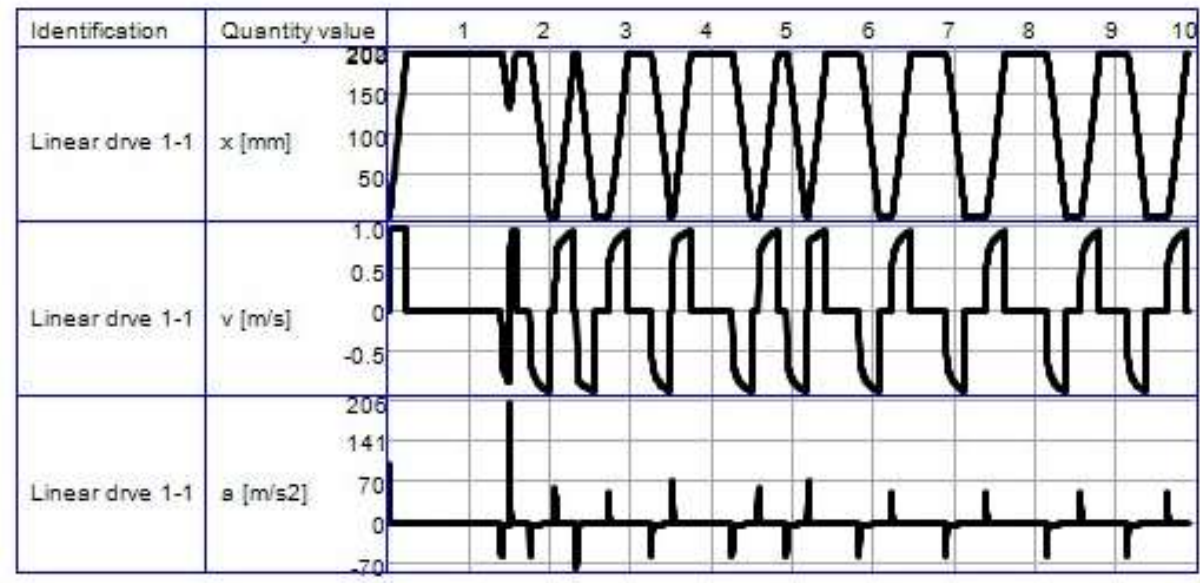

Figure 7. Diagrams of functional parameters variations of the linear drive

In the table 2 below there are given the component devices used in the second pneumatic scheme.

\begin{tabular}{l|c}
\hline \multicolumn{1}{c|}{ Description } & Number of components \\
\hline Compressed air supply & 1 \\
\hline Air service unit & 1 \\
\hline 5/2 way solenoid valve with spring & 4 \\
\hline Throttle valve & 1 \\
\hline Linear drive with shape-fitting adaptor & 1 \\
\hline Linear drive with solenoid coupling & 1 \\
\hline
\end{tabular}

Table 2. Components of the second pneumatic circuit.

The second pneumatic circuits studied uses two actuators: linear drive with shape-fitting adaptor (2-1) and linear drive with solenoid coupling (2-2), Figure 8.

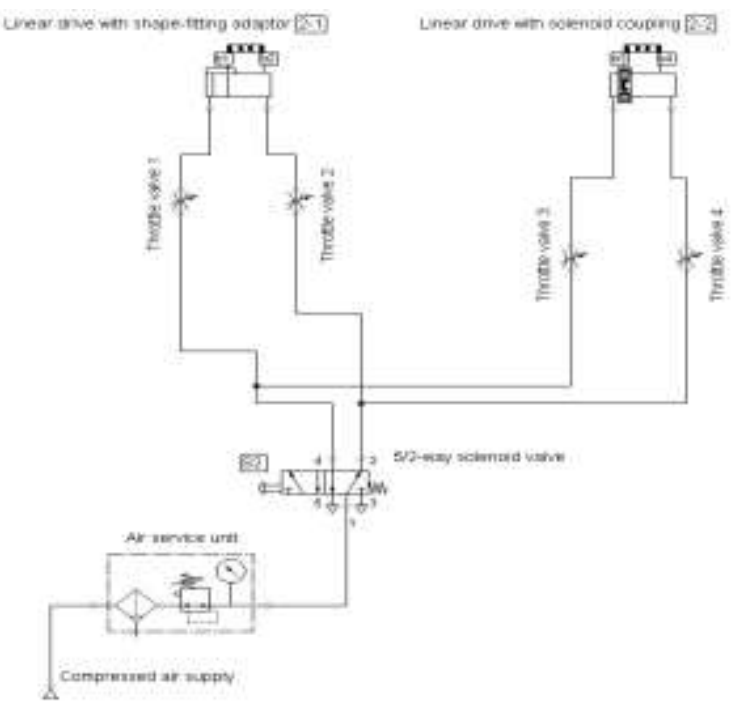

Figure 8. Second pneumatic circuit using two linear drives 
In this case, the operator presses button $\mathrm{S} 2$ and the linear drives open together. This means, the piston of linear drive with shape-fitting adaptor (2-1) moves from point b1 to point $b 2$, respectively the piston of linear drive with solenoid coupling (2-2) moves from point $b 3$ to point $b 4$. After that, both pistons return to their starting points, because the $5 / 2$ way solenoid valve has a spring, Figure 9.

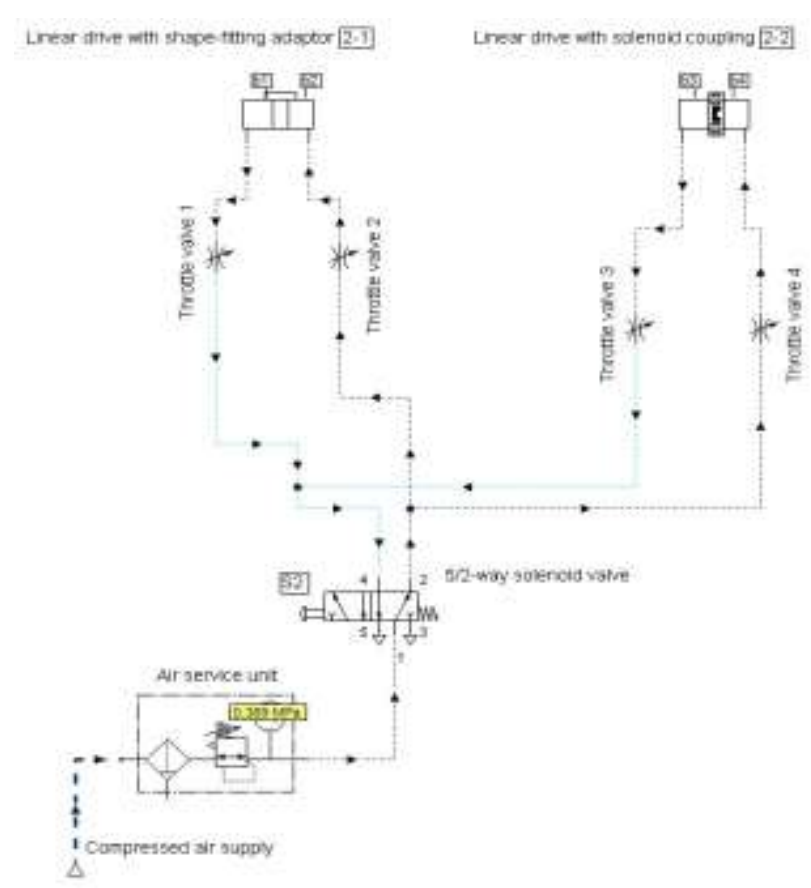

Figure 9: Simulation of the second pneumatic circuit

\section{The electro-pneumatic circuits with linear actuator}

Electro-pneumatic circuits using linear actuators are more complex than the pneumatic ones using as devices the same linear drive, [6].

Comparison between the pneumatic scheme and the electro-pneumatic scheme essentially demonstrates following advantages:

- The circuit allows easy automation of complex industrial processes;

- Electric equipment costs less than pneumatic equipment;

- The loading gauge of the control equipment is reduced.
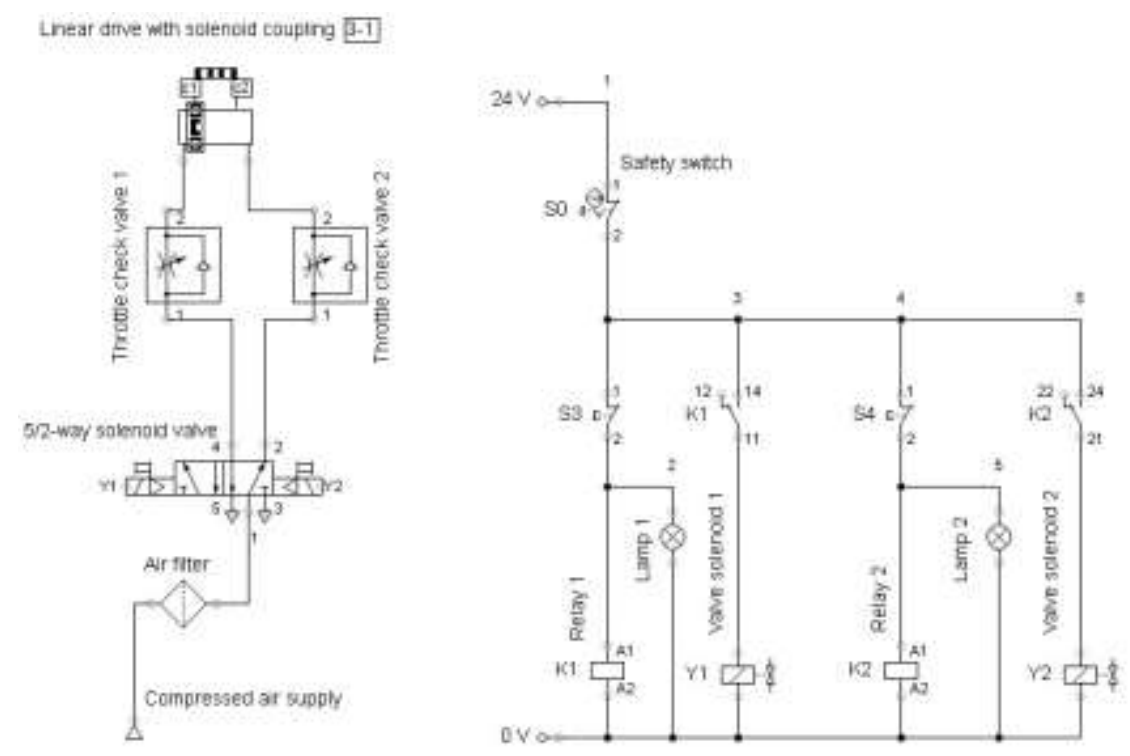

Figure 10: First electro-pneumatic circuit 
The first electro-pneumatic circuit comprises a few basic pneumatic and electrical devices. It must be noted that the authors used a latched 5/2 - way solenoid valve, having a "memory". This "memory" has an effect on commands given by the operator of this installation, Figure 10.

In the table 3 below there are given the component devices used in the first electro-pneumatic circuit.

\begin{tabular}{l|c}
\hline \multicolumn{1}{c|}{ Description } & Number of components \\
\hline Compressed air supply & 1 \\
\hline Air filter & 1 \\
\hline $5 / 2$ way solenoid valve with spring & 1 \\
\hline Throttle valve & 2 \\
\hline Linear drive with solenoid coupling & 1 \\
\hline Relay & 2 \\
\hline Valve solenoid & 2 \\
\hline Lamp & 2 \\
\hline
\end{tabular}

Table 3. Devices in the first electro-pneumatic scheme.

In order to operate the electro-pneumatic scheme shown in figure 11, having one linear drive with solenoid coupling (3-1), operator has to press S3 button. Then, the piston of linear drive moves from point $\mathrm{c} 1$ to point $\mathrm{c} 2$ and lamp 1 shows a yellow signal, Figure 11.
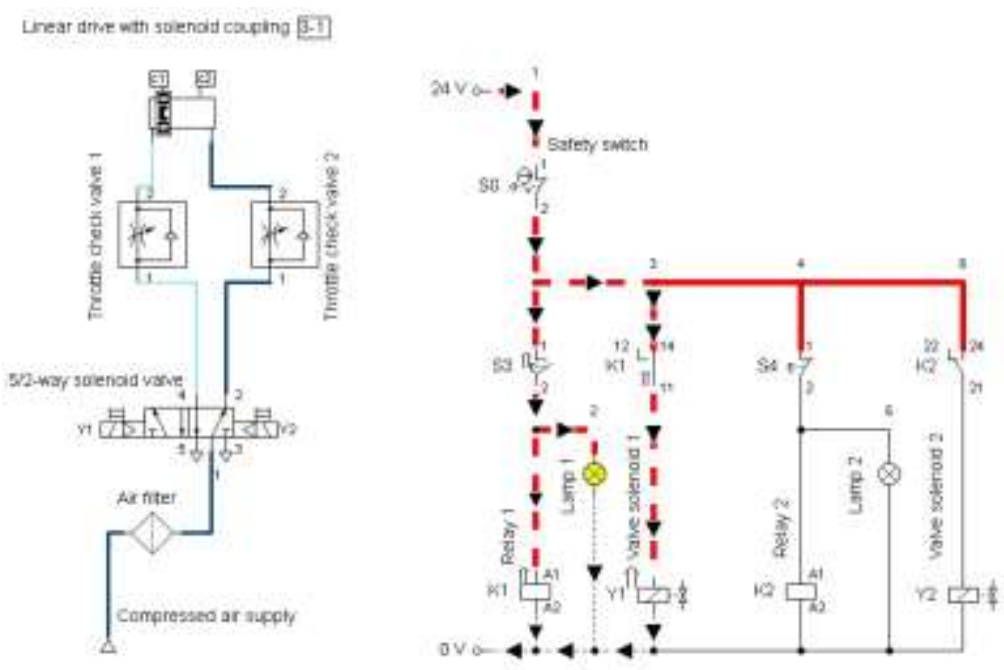

Figure 11. Opening the first electro-pneumatic circuit

In order to close the first electro-pneumatic scheme, operator must press button S4. The piston of linear drive moves now from point $\mathrm{c} 2$ to point $\mathrm{c} 1$ and lamp 2 shows a yellow signal, Figure 12.
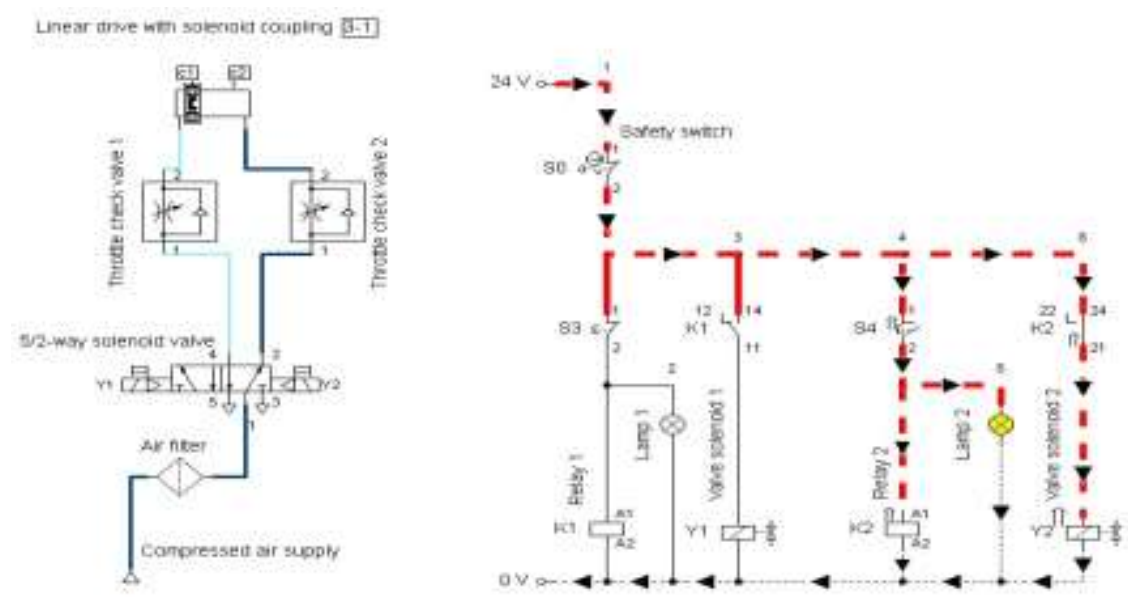

Figure 12. Closing the first electro-pneumatic circuit. Simulation 
Another further improvement for the scheme presented above is the use of a programmable logic controller (PLC), providing much more flexibility in controlling the linear drive, using a shape-fitting adaptor (4-1), Figure 13.
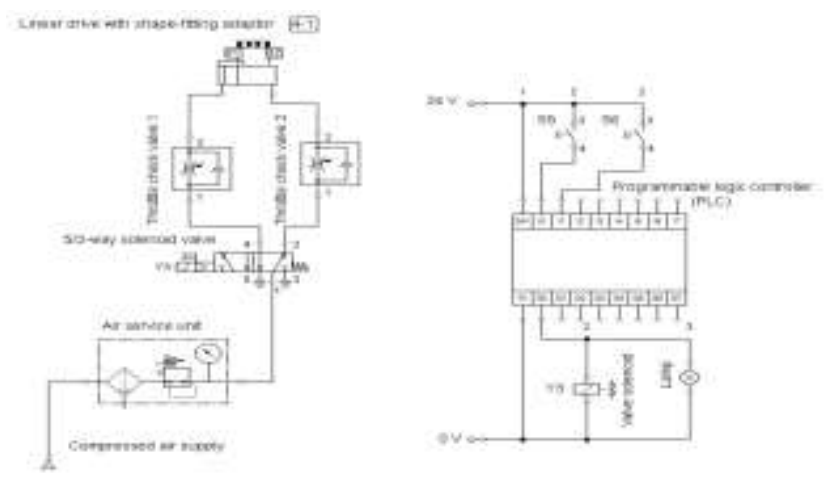

Figure 13. Second electro-pneumatic circuit using a PLC

Another further improvement for the scheme presented above is the use of a programmable logic controller (PLC), providing much more flexibility in controlling the linear drive, using a shape-fitting adaptor (4-1), Figure 13.

In table 4 below are given some important actuators used in the second electro-pneumatic circuit, [7].

\begin{tabular}{l|c}
\hline \multicolumn{1}{c|}{ Description } & Number of components \\
\hline Compressed air supply & 1 \\
\hline Air service unit & 1 \\
\hline 5/2 way solenoid valve with spring & 1 \\
\hline Throttle valve & 4 \\
\hline Linear drive with shape-fitting adaptor & 1 \\
\hline Relay & 1 \\
\hline Valve solenoid & 1 \\
\hline Lamp & 1 \\
\hline Programmable logic controller (PLC) & 1 \\
\hline
\end{tabular}

Table 4. Devices in the first electro-pneumatic scheme.

The next two figures show the movement of the linear drive with shape-fitting adaptor (4-1). Thus, in order to open the second electro-pneumatic scheme, operator must press S5 button. In this case, the piston of linear drive with shape-fitting adaptor (4-1) moves from point $\mathrm{d} 1$ to point $\mathrm{d} 2$ and lamp shows a yellow signal, Figure 14.
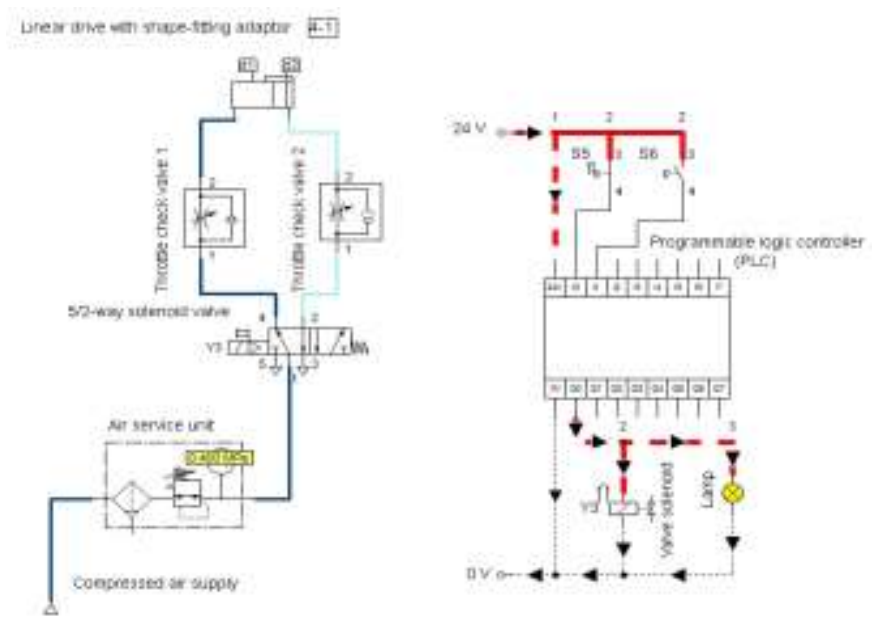

Figure 14. Opening the second electro-pneumatic circuit using a PLC. Simulation 
However, in order to close the first electro-pneumatic scheme, operator must press S6 button. Therefore, the piston of linear drive moves from point $\mathrm{d} 2$ to point $\mathrm{d} 1$, Figure 15.
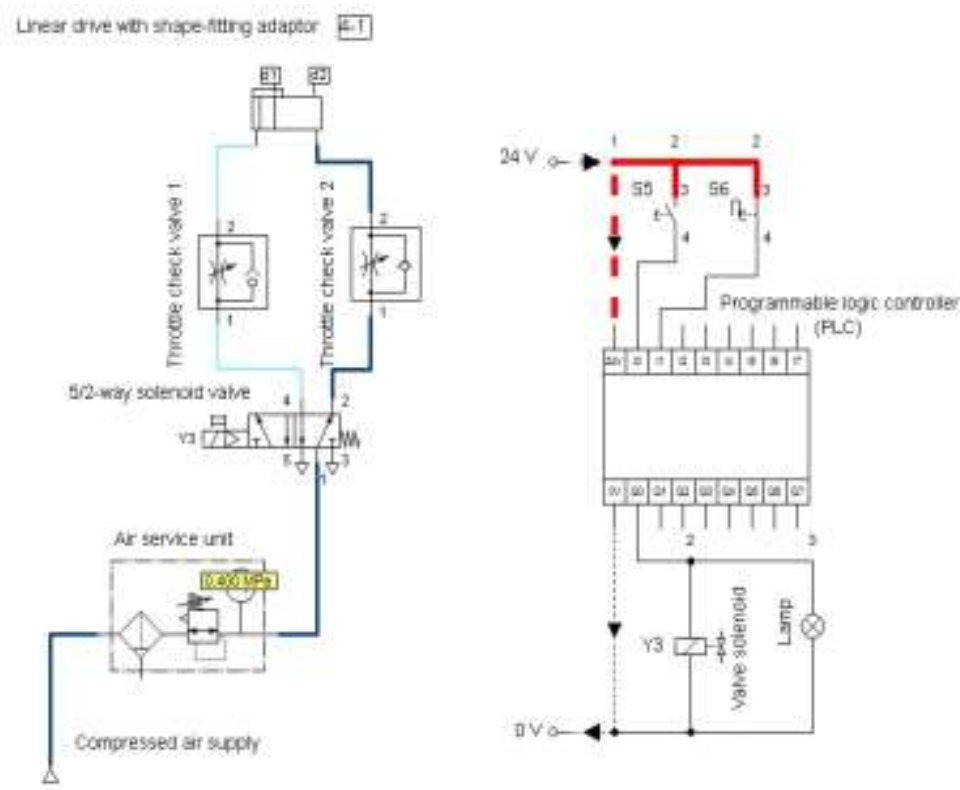

Figure 15. Closing the second electro-pneumatic circuit using a PLC. Simulation.

\section{Conclusions}

Taking into consideration what has been presented in this paper, the electro-pneumatic schemes with linear drives offer some important advantages over traditional electro-pneumatic circuits using other actuators (ex. cylinder, fluidic muscle, semi-rotary, etc.). These advantages are: higher power to weight ratio, lower cost, less weight, dust no stiction, permeability to dirt, etc.

Given their superior strength, it seems the linear drive will replace in time, in some applications, the pneumatic cylinders from electro-pneumatic installations.

Because each product development generates its own individual engineering challenges, it would be however, a nonsense to claim that linear device could be a complete substitute for a pneumatic actuator from electro-pneumatic installations.

These actuators also offer some unique features, which are to be explored in a wide variety of fields: agriculture, defence, industry, etc.

Users and researchers should be encouraged to take into account the linear drive when searching for a suitable fluid movement device in electro-pneumatic installations.

Further research involving this pneumatic device is to be encouraged, including those that would investigate the possibility of fluid muscles in electro-pneumatic circuits using logic modules.

A more sophisticated electro-pneumatic scheme with linear drives and programmable logic controller (PLC) is to be developed in future papers.

\section{Acknowledgement}

This work was supported by:

- A grant of the Romanian Ministry of Education and Research, CCCDI - UEFISCDI, project number PN-III-P2.1-PTE-2019-0446, within PNCDI III, funding contract no. 53PTE/23.09.2020, project title "Hydrophilic auto-chassis for high energy efficiency operation of interchangeable equipment intended for performing public utility work", acronym ASHEUP, research direction 3.Econano-technologies and advanced materials, subdomain Advanced materials;

- a grant of the Romanian Ministry of Education and Research, CCCDI - UEFISCDI, project number PNIII-P2.1-PTE-2019-4123, within PNCDI III, funding contract no. 380PED/23.10.2020, project title "Multifunctional hexa-rotors tricopter for precision farming environment", acronym 4.0-MHRT, project domain 1.Bioeconomics, subdomain 1.1. 


\section{References}

[1] ALBOIU, E.F., DEGERATU, M., ALBOIU, N.I., Mathematical modelling of the processes occurring in gas mixtures used in hyperbaric facilities, Sustainable Solutions for Energy and Environment, EENVIRO-YRC, Bucharest, Romania, 2015.

[2] AVRAN, M., NITU, C., GRAMESCU, B., Linear pneumatic actuator, MATEC Web of Conferences 121:08001, doi: 10.1051/matecconf/201712108001, 2017.

[3] NĂSTĂSESCU, V., BÂRSAN, Gh., Metoda elementelor libere Galerkin în analiza strucurilor, Ed. Academiei Române, București, 2018.

[4] DUMITRACHE, C.L., DELEANU, D., Computational NX Fluid Structure Interaction (FSI) analysis on naval three way ball valve, Conference Series: Materials Science and Engineering, ModTech, doi: 10.1088/1757-899X/1182/1/012021, 2021.

[5] PANAITESCU, M., PANAITESCU, F.V., Optimizing Air Flow Instilled in an Aeration Pool, Journal Hidraulica, no. 4, pp. 72-75, Bucharest, Romania, 2018.

[6] SURDU, G., Sustainable development a challenge for research in the field military equipment and space, Impact of Socio-economic and Technological Transformations at National, European and International level (ISETT), vol. 7, ED. Institute for World Economy, Romanian Academy, 2015.

[7] RAMIREZ, I. (2018). Design of a tracking controller of a siso system of pneumatic servopositioning, Ing. Dessaro, vol. 36, no. 1, doi: 10.14482/inde.36.1.10940. 